\title{
A Simple Model of Radiation from a Magnetized Neutron Star: Accreted Matter and Polar Hotspots
}

\author{
Dmitry Yakovlev (D)
}

Ioffe Institute, Politekhnicheskaya 26, 194021 St. Petersburg, Russia; yak.astro@mail.ioffe.ru

\begin{abstract}
A simple and well known model for thermal radiation spectra from a magnetized neutron star is further studied. The model assumes that the star is internally isothermal and possesses a dipole magnetic field $\left(B \lesssim 10^{14} \mathrm{G}\right.$ ) in the outer heat-insulating layer. The heat transport through this layer makes the surface temperature distribution anisotropic; any local surface element is assumed to emit a blackbody (BB) radiation with a local effective temperature. It is shown that this thermal emission is nearly independent of the chemical composition of insulating envelope (at the same taken averaged effective surface temperature). Adding a slight extra heating of magnetic poles allows one to be qualitatively consistent with observations of some isolated neutron stars.
\end{abstract}

Keywords: neutron stars; radiation transfer; magnetic fields

Citation: Yakovlev, D. A Simple Model of Radiation from a Magnetized Neutron Star: Accreted Matter and Polar Hotspots. Universe 2021, 7, 395. https://doi.org/ 10.3390/universe7110395

Academic Editors: Nazar R. Ikhsanov, Galina L. Klimchitskaya and Vladimir M. Mostepanenko

Received: 30 September 2021

Accepted: 18 October 2021

Published: 21 October 2021

Publisher's Note: MDPI stays neutral with regard to jurisdictional claims in published maps and institutional affiliations.

Copyright: (C) 2021 by the author. Licensee MDPI, Basel, Switzerland. This article is an open access article distributed under the terms and conditions of the Creative Commons Attribution (CC BY) license (https:/ / creativecommons.org/licenses/by/ $4.0 /)$.

\section{Yury N. Gnedin}

This paper is dedicated to the memory of Yury N. Gnedin (1935-2018) Figure 1. He was my senior colleague at Theoretical Astrophysics Department of Ioffe Insitute from 1971 to 1984, and we often met later, when he moved to Pulkovo Observatory. He was always interested in many scientific fields. For instance, his pet subjects were radiation transfer, neutron stars and magnetic fields (although he never lost scientific interest in a great amount of other things). When neutron stars were discovered in 1967, he was extremely enthusiastic about them and initiated ultrafast spreading of interest to these objects among colleagues. He was the first author of the first publication [1] on neutron stars at the Ioffe Institute after the discovery of these stars. He wrote-with different co-authors-plenty of (now) classical papers on neutron star physics. In particular, they include a basic formulation of the radiation transfer problem in a strongly magnetized plasma [2], a theoretical prediction of electron cyclotron lines in the spectra of radiation from magnetized neutron stars [3], the first realistic studies of ionization in the magnetized hydrogen atmospheres of neutron stars [4]. He was also greatly fond of history.

Although we did not work much together, I, as many others, respected Yury Gnedin very much. When he could help, he really did, and he was a Real Gentleman-friendly and open to any one, from a prominent scientist to a hopeless student. I will always remember the wonderful atmosphere, created by Yury Gnedin, and warm hospitality of his family and home. It is amazing that both his sons have grown up into excellent scientists.

I present a small piece of neutron star physics on thermal radiation from magnetized neutron stars. Hopefully, he would like it. 

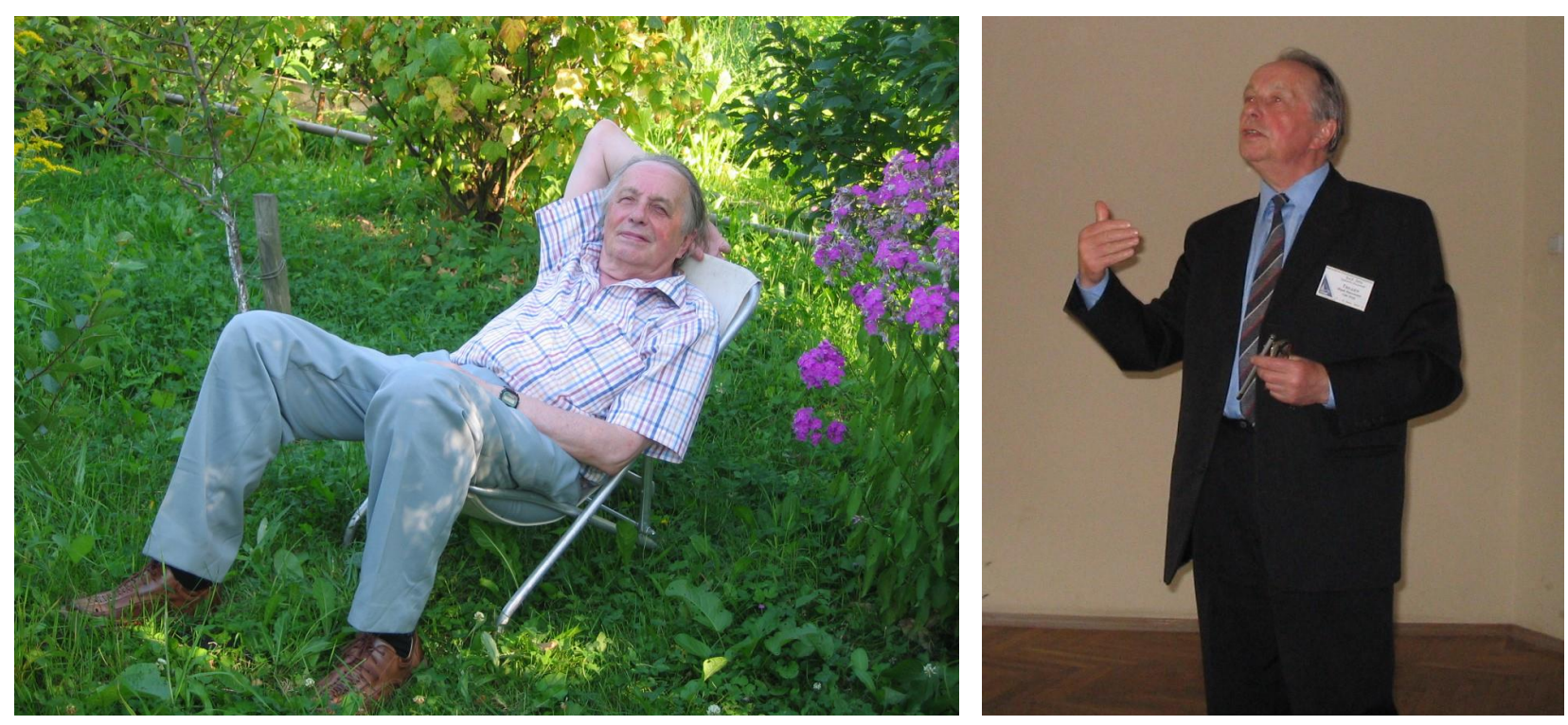

Figure 1. Yury N. Gnedin. (Left): A rare moment of leisure on his birthday, 13 August 2007, near his summer cottage. (Right): At a conference (June 2004). Courtesy to Oleg Gnedin.

\section{Introduction}

I will mostly consider thermal X-ray radiation from the surface of a middle-aged $\left(t \lesssim 10^{6} \mathrm{yr}\right)$ isolated magnetized neutron star. The radiation is supposed to emerge from the warm neutron star interior and is emitted from a thin atmosphere or condensed surface (e.g., [5]), where the radiation spectrum is formed. The interiors of the star are thought to be nearly isothermal because of the high thermal conductivity of dense matter. Nevertheless, the interiors are thermally insulated from the surface by a thin heat blanketing envelope, where the thermal conduction is poorer and more sensitive to the chemical composition and magnetic field. The field redirects the emerging heat flow and results in anisotropic surface temperature distribution. The thermal surface emission can also become anisotropic.

The anisotropy of observed surface radiation is used to infer magnetic field strength and geometry, the composition of the surface layers, global parameters of the star (such as mass and radius), as well as important parameters of superdense matter in stellar interiors (see, e.g., [5,6] and references therein).

Here, I study a simple model of thermal emission from magnetized neutron stars with isothermal interiors, assuming the star emits a blackbody (BB) radiation with a local effective surface temperature $T_{\mathrm{s}}$, which varies over the stellar surface under the effects of dipole surface magnetic field. This model has been invented long ago by Greenstein and Hartke [7], elaborated in the literature (e.g., [8-13]) and reviewed in [6,14]. Here I point out some properties of the surface emission, which, to the best of my knowledge, have not been studied in the literature.

Logically, this paper continues the previous one [15] (hereafter Paper I), which shows that the simple thermal spectra of magnetized neutron stars can be accurately approximated by two-BB (2BB) models. Section 3 outlines theoretical formalism. Section 4 is devoted to the effects of chemical composition of the blanketing envelopes and Section 5 extends the solution to the case, in which magnetic poles contain additional hot spots. The conclusions are formulated in Section 6.

It is important to mention more complicated neutron star emission models. They include sophisticated magnetic field effects on thermal emission, leading to specific spectral, polarization and angular properties of radiation (see, e.g., [6,16-18]), which are not reproduced by the given model. 


\section{Simple Model Spectra}

\subsection{General Formalism}

The calculation of radiation spectral flux from a spherical neutron star under formulated assumptions is simple. Here is the summary using the notations of Paper I. Any small surface temperature element emits like a BB, and the flux is obtained by intergating over a visible part of the surface, taking into account that emitted quanta propagate in Schwarzschild space-time and demonstrate the gravitational redshift of photon energies and light bending. The effects of General Relativity are specified by the compactness parameter $x_{\mathrm{g}}=r_{\mathrm{g}} / R$, where $r_{\mathrm{g}}=2 G M / c^{2}$ is the Schwartschield radius, $M$ is the gravitational star's mass, $R$ is the circumferential radius, $G$ is the gravitational constant and $c$ is the velocity of light. One deals either with local quantities at the stellar surface (e.g., the local surface temperature $T_{\mathrm{s}}$ ) or with the quantities detected by a distant observer. The letter ones will often be marked by the symbol ' $\infty$ '. For instance, $T_{\mathrm{s}}^{\infty}=T_{\mathrm{s}} \sqrt{1-x_{\mathrm{g}}}$ is the redshifted surface temperature. As an exclusion, we will denote local (non-redshifted) photon energy by $E_{0}$, and the redshifted energy by $E \equiv E_{\infty}=E_{0} \sqrt{1-x_{\mathrm{g}}}$.

Let $F_{E}^{\infty}$ be a radiative spectral flux density $\left[\mathrm{erg} \mathrm{cm}^{-2} \mathrm{~s}^{-1} \mathrm{keV}^{-1}\right.$ ] detected at a distance $D \gg R$. It is customary to express $F_{E}^{\infty}$ as:

$$
F_{E}^{\infty}=\frac{R^{2}}{D^{2}} H_{E}^{\infty},
$$

where $H_{E}^{\infty}$ is the effective flux, that is formally independent of $D$. It can be calculated as an integral of the radiating surface flux over the visible part of the surface,

$$
H_{E}^{\infty}=\frac{15 \sigma_{\mathrm{SB}}}{16 \pi^{5} k_{\mathrm{B}}^{4}} \int_{\mathrm{viz}} \mathrm{d} \Omega_{\mathrm{s}} \frac{\left(1-x_{\mathrm{g}}\right)^{-1} \mathcal{P} E^{3}}{\exp \left(E / k_{\mathrm{B}} T_{\mathrm{s}}^{\infty}\right)-1}
$$

where $\sigma_{\mathrm{SB}}$ is the Stefan-Boltzmann constant, $k_{\mathrm{B}}$ is the Boltzmann constant, $\mathrm{d} \Omega_{\mathrm{S}}$ is a surface solid angle element, and $\mathcal{P}$ is the light bending function (e.g., [5,19-21]).

It is convenient to integrate over the star's surface and calculate the bolometric luminosity of the star, $L_{\mathrm{S}}$, as well as the averaged non-redshifted effective surface temperature $T_{\text {eff }}$ (e.g., [10]),

$$
L_{\mathrm{s}}=\sigma_{\mathrm{SB}} R^{2} \int_{4 \pi} \mathrm{d} \Omega_{\mathrm{s}} T_{\mathrm{s}}^{4} \equiv 4 \pi \sigma_{\mathrm{SB}} R^{2} T_{\text {eff }}^{4} .
$$

For a uniform surface temperature $\left(T_{\mathrm{s}}=T_{\mathrm{eff}}\right)$, one immediately gets the standard BB flux,

$$
H_{E}^{\mathrm{BB} \infty}=\frac{15 \sigma_{\mathrm{SB}}}{4 \pi^{4} k_{\mathrm{B}}^{4}} \frac{\left(1-x_{\mathrm{g}}\right)^{-1} E^{3}}{\exp \left(E / k_{\mathrm{B}} T_{\mathrm{eff}}^{\infty}\right)-1},
$$

and the bolometric effective flux,

$$
H_{\mathrm{bol}}^{\mathrm{BB} \infty}=\int_{0}^{\infty} H_{E}^{\mathrm{BB} \infty} \mathrm{d} E=\frac{\sigma_{\mathrm{SB}} T_{\mathrm{eff}}^{\infty 4}}{1-x_{\mathrm{g}}} .
$$

\subsection{Input Parameters}

Equation (2) allows one to compute thermal spectra for any given temperature distribution $T_{\mathrm{S}}$ over the neutron star surface. We focus on the distribution created by a dipole magnetic field (with the field strength $B_{\text {pole }}$ at magnetic poles) due to anisotropic heat transport in a thin (maximum a few hundreds meters) heat blanketing envelope. The input parameters are $M, R$, chemical composition of the blanketing envelope, and a nonredshifted temperature $T_{\mathrm{b}}$ at its bottom (at density $\rho_{\mathrm{b}} \sim 10^{10} \mathrm{~g} \mathrm{~cm}^{-3}$; see, for example, [14]). The local $T_{\mathrm{s}}$ is usually determined by solving local quasistationary radial heat transport within the envelope mediated by an effective radial thermal conductivity. For studying the thermal surface emission, it is profitable to use $T_{\text {eff }}$ [see Equation (3)] instead of $T_{\mathrm{b}}$. 


\section{3. $2 B B$ Representation}

According to Paper I, the spectral fluxes $H_{E}^{\infty}$, computed from Equation (2) for iron heat blankets, are accurately fitted by a familiar 2BB model,

$$
H_{E}^{\infty}=s_{\mathrm{c}} H_{E}^{\mathrm{BB} \infty}\left(T_{\text {effc }}\right)+s_{\mathrm{h}} H_{E}^{\mathrm{BB} \infty}\left(T_{\text {effh }}\right) .
$$

Here, $H_{E}^{\mathrm{BB} \infty}\left(T_{\text {eff }}\right)$ is given by Equation (4); ' $c$ ' and ' $\mathrm{h}$ ' refer, respectively, to colder and hotter $\mathrm{BB}$ components. Any fit contains four parameters, which are two effective temperatures $T_{\text {effc }}$ and $T_{\text {effh }}$, and two fractions of effective radiating surface areas, $s_{\mathrm{c}}$ and $s_{\mathrm{h}}$. Instead of $T_{\mathrm{effc}}$ and $T_{\mathrm{effh}}$, it is convenient to introduce two dimensionless parameters $p_{\mathrm{c}}=T_{\text {effc }} / T_{\text {eff }}$ and $p_{\mathrm{h}}=T_{\text {effh }} / T_{\text {eff }}$, with $p_{\mathrm{c}}<p_{\mathrm{h}}$. In Paper I the fits have been done for a number of representative values of $M, R, \log T_{\text {eff }}[\mathrm{K}]$ (from 5.5 to 6.8), $\log B_{\text {pole }}[\mathrm{G}]$ (from 11 to 14$)$, photon energies $(0.064<E \lesssim 40 \mathrm{keV}$, removing those $E$ at which the fluxes are negligibly small) and inclination angles $i$ (between line of sight and the magnetic axis). Typical relative fit errors have not exceeded a few percent, meaning the fits were very good, providing excellent analytic representation of the original computed data. Therefore, thermal X-ray spectral fluxes of magnetized neutron stars within the given model are nearly identical to those of 2BB spectral models.

Moreover, as shown in Paper I, it is sufficient to calculate the fluxes $H_{E}^{\infty}$ for two observation directions which are (i) pole observation, $i=0$, to be denoted as $H_{E}^{\| \infty}$, and (ii) equator observations, $i=90^{\circ}$, to be denoted as $H_{E}^{\perp \infty}$. If these fluxes are known, the radiation flux in any direction $i$ is accurately approximated as:

$$
H_{E}^{i \infty}=H_{E}^{\| \infty} \cos ^{2} i+H_{E}^{\perp \infty} \sin ^{2} i .
$$

We will see (Section 5) that this approximation is more restrictive than Equation (6).

This accurate representation of numerically calculated $H_{E}^{i \infty}$ by a map of four fit parameters $\left(p_{\mathrm{c}}, p_{\mathrm{h}}, s_{\mathrm{c}}, s_{\mathrm{h}}\right)$ in the space of input parameters has to be taken with the grain of salt. The problem is that the fluxes $H_{E}^{i \infty}$ are close to 1BB fluxes (4), which leads to some degeneracy of fit parameters (with respect to a fit procedure and a choice of grid points). On the other hand, the assumed surface temperature distribution model and the local BB emission model are definitely approximate by themselves, so that a too-accurate fitting of $H_{E}^{i \infty}$ is actually purely academic. However, these results can be helpful for the interpretation of observations, especially because the $2 \mathrm{BB}$ model is often used by observers.

\section{Iron and Accreted Heat Blankets}

Paper I considered heat blankets made of iron. Here, we employ a more general model $[10,11,22]$, in which a heat blanket consists of shells (from top to bottom) of hydrogen, helium, carbon and iron. Light elements $(\mathrm{H}, \mathrm{He}, \mathrm{C})$ are thought to appear because of accretion of $\mathrm{H}$ and/or He and further burning into $\mathrm{C}$. Iron is either formed at neutron star birth or is a final result of carbon burning. The mass of lighter elements $\Delta M$ in the heat blanket is treated as a free parameter. The heat blanketing envelope may fully consist of iron (Paper I) or contain some mass $\Delta M \lesssim 10^{-6} M_{\odot}$ of lighter elements. Lighter elements affect the surface temperature distribution and thermal surface spectral fluxes.

To simplify the task, we will study fully accreted blankets and conclude on the partly accreted ones in the end of Section 4.2.

\section{1. $T_{s}$-Distributions}

Our surface temperature distribution $T_{\mathrm{S}}$ is axially symmetric and symmetric with respect to the magnetic equator. For illustration, Figure 2 presents calculated $T_{\mathrm{S}}$ for a star with $M=1.4 M_{\odot}$ and $R=12 \mathrm{~km}\left[x_{\mathrm{g}}=0.344\right.$ and the surface gravity $g_{\mathrm{s}}=G M /\left(R^{2} \sqrt{1-x_{\mathrm{g}}}\right)=$ $1.59 \times 10^{14} \mathrm{~cm} \mathrm{~s}^{-2}$ ]. The effective surface temperatures are shown versus colatitude $\vartheta$, which varies from $\vartheta=0$ at the north pole, to $\vartheta=90^{\circ}$ at the magnetic equator, and then to $\vartheta=180^{\circ}$ at the south pole. Three panels $(\mathrm{a}-\mathrm{c})$ correspond to three values of the magnetic 
field $B_{\text {pole }}=10^{12}, 10^{13}$ and $10^{14} \mathrm{G}$ at the pole. Each panel displays $T_{\mathrm{s}}(\vartheta)$ for six values of $\log T_{\text {eff }}[\mathrm{K}]=5.8,6.0, \ldots 6.8$. Solid lines are plotted for the heat blankets made of iron, while dashed lines are for the fully accreted blankets. The iron and accreted matter have different thermal conductivities and, hence, different $T_{\mathrm{s}}(\vartheta)$ distributions at the same $T_{\text {eff. }}$. The dotted lines correspond to a non-magnetic star to guide the eye (they give $T_{\mathrm{s}}=T_{\text {eff }}$ ).
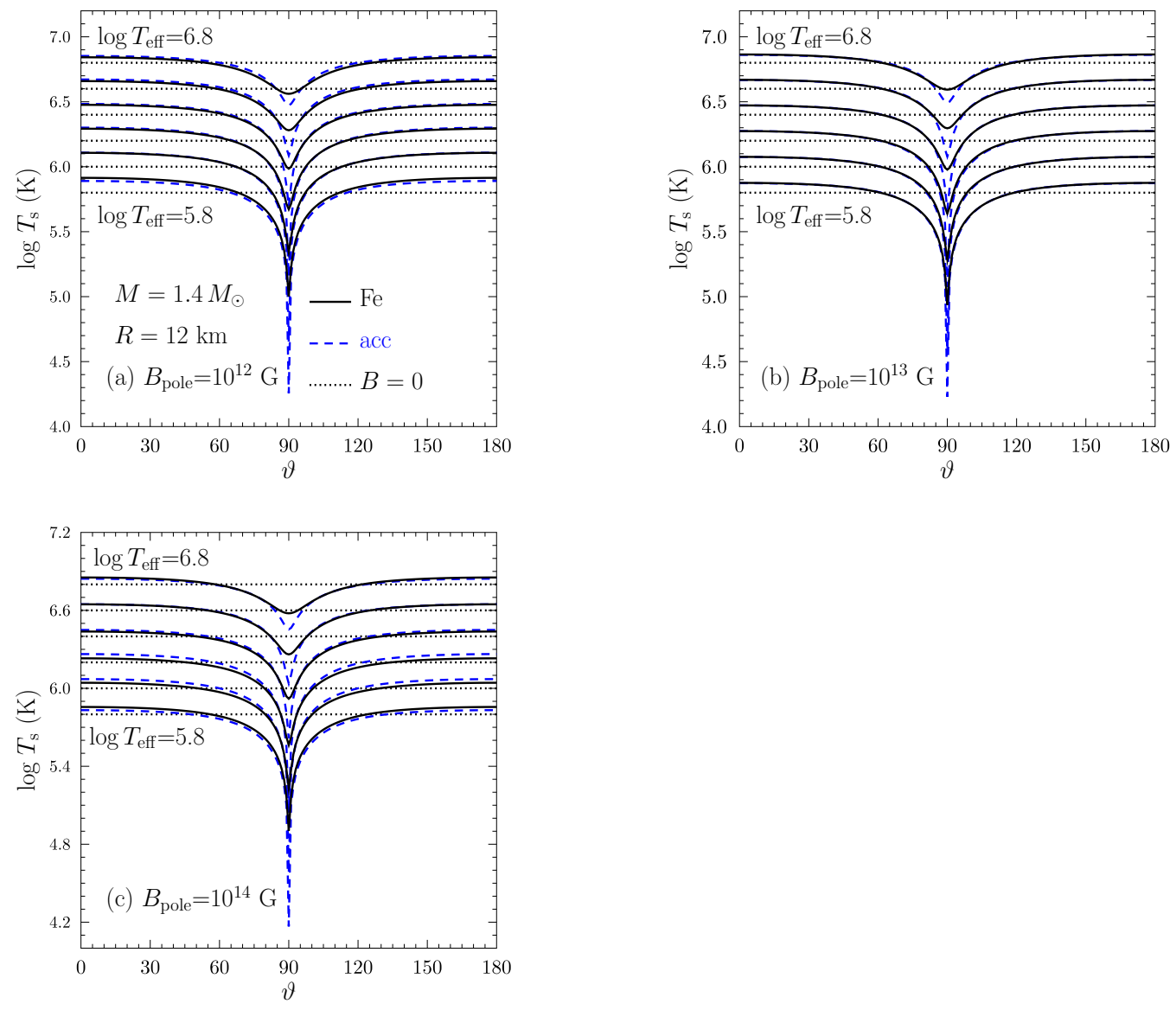

Figure 2. Effective surface temperature $T_{\mathrm{S}}$ versus colatitude $\vartheta$, from magnetic pole $(\vartheta=0)$ to equator $\left(\vartheta=90^{\circ}\right)$ and the opposite magnetic pole $\left(\vartheta=180^{\circ}\right)$ of a neutron star with $M=1.4 M_{\odot}$ and $R=12 \mathrm{~km}$ at three values of $B_{\text {pole }}=10^{12}, 10^{13}$ and $10^{14} \mathrm{G}$ [panels (a-c)]. The curves are plotted for six effective surface temperatures, $\log T_{\text {eff }}[K]=5.8,6.0 \ldots 6.8$. Solid curves refer to the heat-blanket model made of iron, dashed cures refer to the heat blanket of fully accreted matter; dotted curves correspond to the field-free star.

Heat conduction is mainly radiative in the outer non-degenerate layer. In deeper layers, where the electrons become degenerate, heat is mostly transported by electrons. The field affects also thermodynamic properties of the matter, for instance, the pressure (e.g., [23]). The magnetic field effects, which regulate the anisotropy of the surface temperature distribution, are twofold.

The effects of the first type are the classical effects of electron rotation about magnetic field lines. They are especially important near the equator, where the heat, emergent from the stellar interiors, propagates mainly across field lines and is thus suppressed. This may strongly enhance thermal insulation of the equatorial part of the heat blanketing envelope, producing rather narrow equatorial dips of $T_{\mathrm{s}}(\vartheta)$ (the cold equatorial belt, Figure 2). The dips for the accreted matter are seen to be much stronger than for the iron matter, especially at lower $T_{\text {eff }}$.

The effects of the second type are associated with the Landau quantization of electron motion across $B$-lines. They become more efficient at higher $B$ near magnetic poles, where 
they make the heat insulating layers more heat transparent and tend to increase $T_{\mathrm{S}}$. These relatively warmer polar 'caps' are wide (Figure 2); $T_{\mathrm{s}}$ increases inside them, if we approach a pole, but not dramatically.

As long as $B_{\text {pole }}$ is rather weak $\left(B_{\text {pole }} \lesssim 10^{11} \mathrm{G}\right)$, the classical effects stay more important, producing a pronounced colder equatorial belt, but weakly affecting the polar zones. The surface becomes overall colder, compared with the field-free star with the same internal temperature $T_{\mathrm{b}}$ (e.g., see Figures 22 and 23 in [14]). With increasing $B_{\text {pole, }}$ the quantum effects become dominant, creating hot polar zones and making the surface overall hotter (at the same $T_{\mathrm{b}}$ ). At $B_{\text {pole }} \gtrsim 10^{12} \mathrm{G}$, the surface becomes hotter than at $B_{\text {pole }}=0$; hot polar zones make the equatorial belt less significant.

It is remarkable (Figure 2) that, outside the equatorial belt, at fixed $T_{\text {eff, }}$, the surface temperature profiles $T_{\mathrm{S}}(\vartheta)$ for iron and accreted heat blankets are mainly close. Moreover, these profiles almost coincide at $B_{\text {pole }} \approx 10^{13} \mathrm{G}$. The latter conclusion is independent of specific values of $M$ and $R$.

\subsection{Spectral Fluxes}

Despite the dramatic interplay of the opposite effects and substantial temperature anisotropy, the surface-integrated spectral fluxes $H_{E}^{\infty}$ of thermal radiation behave smoothly under variations of $B_{\text {pole }}$ and/or $T_{\text {eff }}$. This is because of strong effects of General Relativity, which smooth out photon propagation from the surface to the distant observer $[8,10,11]$. As noted in the cited papers, General Relativity 'hides' magnetic effects on thermal surface emission. Very large magnetic fields, with $B_{\text {pole }} \gtrsim 10^{14}$ (typical of magnetars), start to affect the surface emission much more strongly, but such fields are not considered here. They deserve a special study. In particular, their heat-insulating envelopes can become thick and the $1 \mathrm{D}$ approach to calculating the heat insulation within them can be questionable.

We have checked that the spectral fluxes, calculated for the accreted envelopes, possess the same properties (Section 3.3) as for the iron envelopes. In particular, the flux remains to be close to that calculated in the 1BB approximation at uniform surface temperature $T_{\mathrm{s}}=T_{\text {eff }}$ (see, e.g., Figure 1 in Paper I). It is disappointing, meaning that such a flux, taken at $T_{\text {eff }}$ as an input parameter, carries little information on the magnetic field. Then, one should study deviations from the 1BB approximation. It is important that if one fixes $T_{\mathrm{b}}$ and varies $B_{\text {pole }}$, one obtains noticeable $T_{\mathrm{s}}$ and flux variations (as demonstrated, for instance, in Figures 23 and 24 of [14]) but these variations are accompanied by variations of $T_{\text {eff }}$. This would be a good theoretical construction, if one knew $T_{b}$. However, the observer can measure $T_{\text {eff }}$ and wishes to infer $T_{\mathrm{b}}$, which is not easy because at fixed $T_{\text {eff }}$, the theoretical spectral flux is slightly sensitive to $T_{\mathrm{b}}$.

Figure 3 shows thermal spectral flux densities $H_{E}^{\| \infty}$ for polar observations of the same star as in Figure 2 (at seven values of $T_{\text {eff }}$ and three values of $B_{\text {pole }}$ ). The fluxes are plotted in logarithmic scale versus the decimal logarithm of the redshifted photon energy $E$. For each value of $T_{\text {eff }}$ we present three curves. The solid curves give the fluxes radiated from the star with iron heat blanket. The dashed curves present similar fluxes for accreted heat blankets. The dotted curves demonstrate the 1BB model, Equation (4), with given constant $T_{\mathrm{s}}=T_{\text {eff }}$ (as if the magnetic field is absent).

All three fluxes for each $T_{\text {eff }}$ look close in the logarithmic format of Figure 3 . However, they do not coincide (as clearly seen from Figure 3 of Paper I). Note that the difference between the fluxes emitted from the star with accreted and iron envelopes is noticeably smaller than the difference between these fluxes and 1BB ones. The difference from 1BB fluxes monotonically increases with growing $B_{\text {pole }}$ and $E$, which is quite understandable. Higher $B_{\text {pole }}$ produces stronger anisotropy of $T_{\mathrm{s}}$ distribution. Radiation at higher energies is predominantly emitted from hotter places of the surface.

Let us remark that at $B_{\text {pole }}=10^{13} \mathrm{G}$ the spectral fluxes from the star with iron and accreted heat blankets are almost indistinguishable (but sufficiently different from the 1BB flux). This is because the $T_{\mathrm{S}}$ distributions for iron and accreted blankets are very close (Figure 2, Section 4.1). They differ only in cold equatorial belts, but the contribution into 
the fluxes from cold narrow belts appears almost negligible (also see Paper I). In contrast, the contribution from hotter surface regions can be important (Section 5). At $B_{\text {pole }}=10^{14} \mathrm{G}$ the situation remains nearly the same as at $10^{13} \mathrm{G}$.
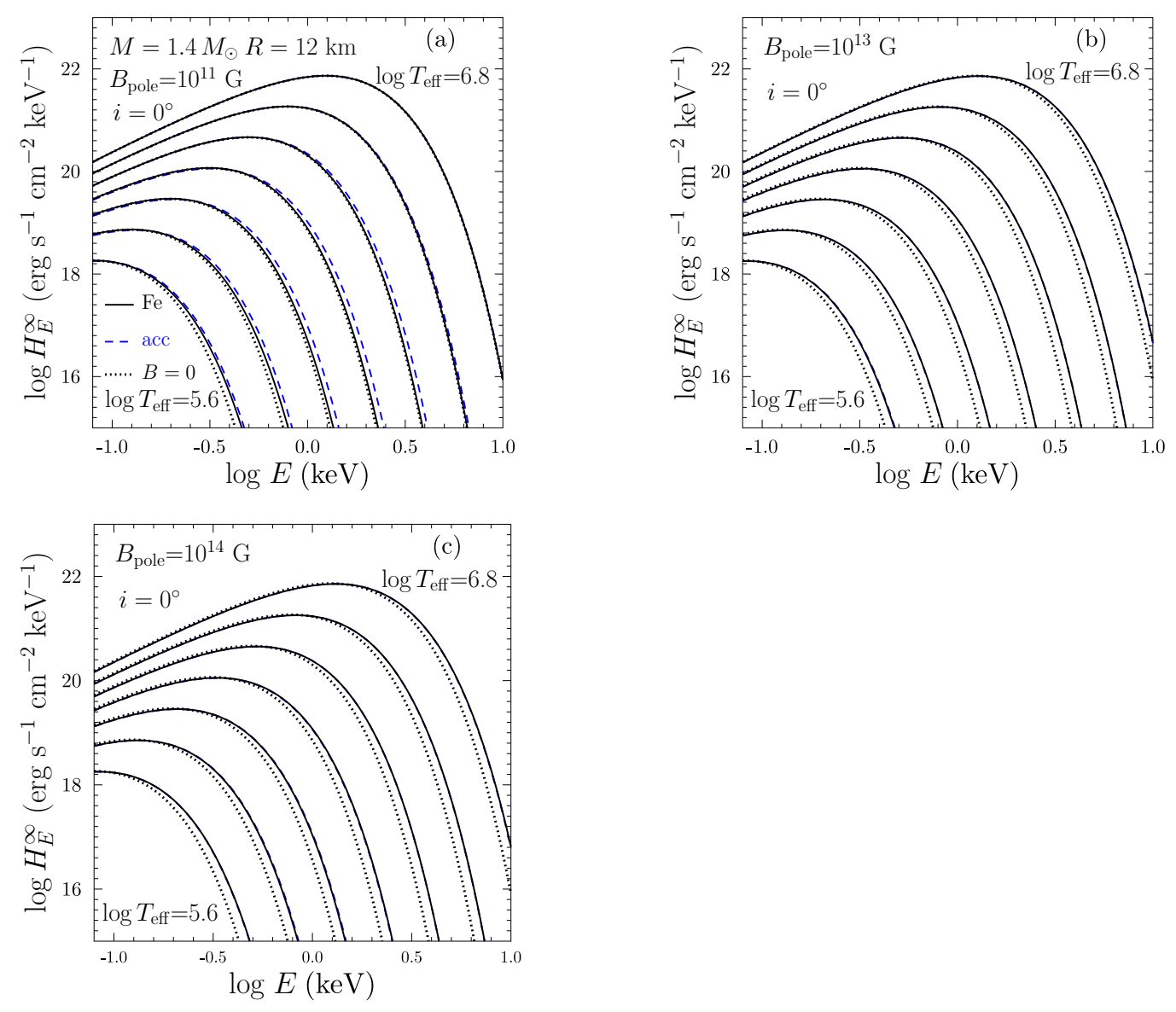

Figure 3. Thermal spectral fluxes observed from magnetic poles for a neutron star with $M=1.4 M_{\odot}$ and $R=12 \mathrm{~km}$ at three values of $B_{\text {pole }}=10^{11} \mathrm{G}(\mathbf{a}), 10^{13} \mathrm{G}(\mathbf{b})$, and $10^{14} \mathrm{G}(\mathbf{c})$ at $\log T_{\text {eff }}=5.6,5.8$, $\ldots, 6.6$ assuming iron (solid lines) or accreted (dashed lines) heat blankets. The dotted lines refer to non-magnetic star to guide the eye.

Figure 4 shows similar spectral fluxes but for equator observations (and only for $B_{\text {pole }}=10^{11}$ and $10^{13} \mathrm{G}$ ). In this case, the situation is similar to that for pole observations.

The main outcome of Figures 3 and 4 is that the difference of the fluxes emitted from stars with iron and accreted heat blanketing envelopes is rather small and can be ignored in many applications, especially taking into account approximate nature of heat blanketing models. The results for partly accreted heat blankets would be similar. Accordingly, one can neglect chemical composition of heat blankets for calculating thermal emission from magnetized neutron stars in the adopted model of heat blankets. Therefore, the chemical composition of heat blankets does affect cooling of the neutron stars but almost does not affect thermal emission (under formulated assumptions). 

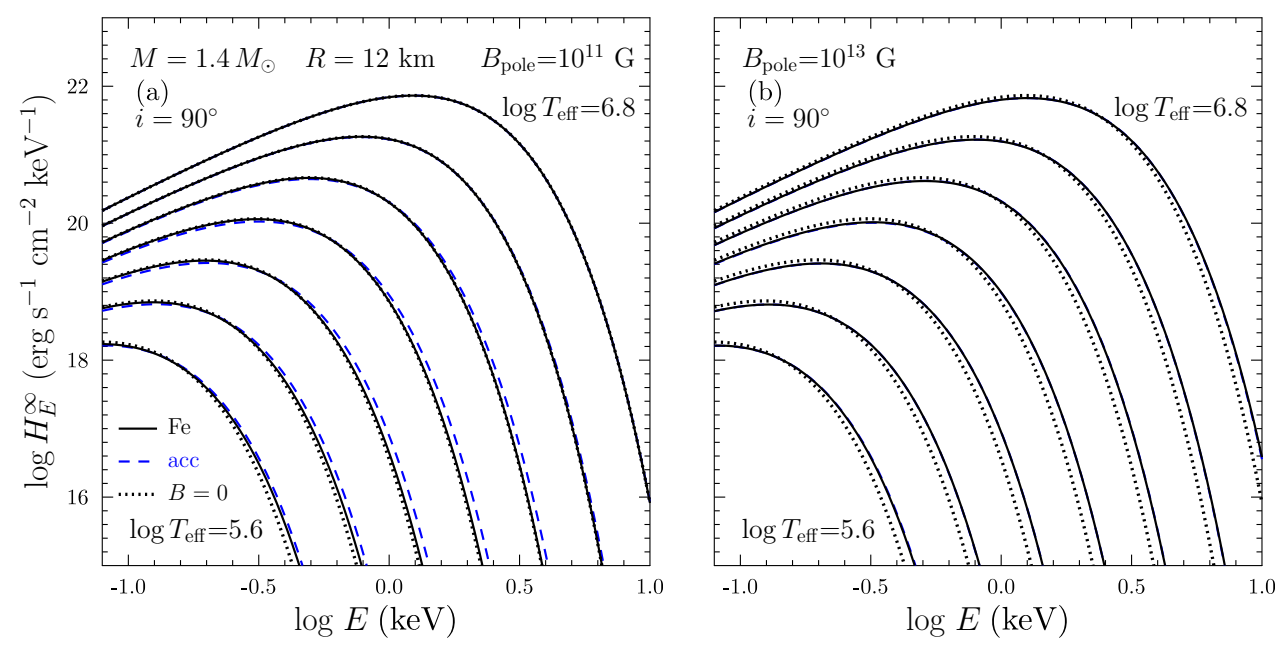

Figure 4. Same as in Figure 3 but for equator observations at $B_{\text {pole }}=10^{11} \mathrm{G}(\mathbf{a})$ and $10^{13} \mathrm{G}(\mathbf{b})$.

\subsection{Phase-Space Maps}

As discussed in Section 3.3 (see also Paper I), thermal spectral fluxes can be presented by maps of fit parameters $\left(p_{\mathrm{c}}, p_{\mathrm{h}}, s_{\mathrm{c}}, s_{\mathrm{h}}\right)$ as functions of input parameters. For completeness, Figure 5 shows these $2 \mathrm{BB}$ maps versus $\log T_{\text {eff }}$ at $B_{\text {pole }}=10^{11} \mathrm{G}$ [panels (a) and (e)], $10^{12} \mathrm{G}$ [panels (b) and (f)], $10^{13} \mathrm{G}[(\mathrm{c})$ and $(\mathrm{g})]$ and $10^{14} \mathrm{G}[(\mathrm{d})$ and (h)]. Panels (a)-(d) refer to pole observations, while (e)-(h) to equator observations. Solid lines correspond to the iron heat blankets, while dashed lines correspond to the accreted ones.

One can see that the dependence of fit parameters on $T_{\text {eff }}$ is smooth, without any specific features. The parameters of both effective BB components are of the same order of magnitude. Most importantly, the temperature $T_{\text {effh }}$ of the hotter BB component exceeds the temperature $T_{\text {effc }}$ of the colder component by less than $20 \%$. The effective surface fraction $s_{\mathrm{c}}$ of the colder component slightly exceeds the fraction $s_{\mathrm{h}}$ of the hotter component.

The maps in Figure 5 represent the same thermal fluxes, which are plotted in Figures 3 and 4. The main conclusion of Section 4.2, based on Figures 3 and 4, is that the fluxes for iron and accreted envelopes are nearly the same. If they were identical, then respective solid and dashed curves in Figure 5 should have been identical as well. However, these curves differ. The difference is especially visible for $B_{\text {pole }}=10^{11}$ and $10^{12} \mathrm{G}$, but less visible at higher $B_{\text {pole }}$. The difference is mainly explained by two things. First, weak variations of 2BB fit parameters lead to weaker variations of the fluxes. Secondly, the $2 \mathrm{BB}$ approximation is accurate but not exact by itself, as discussed in the previous section.

It is important that we fit unabsorbed calculated spectral fluxes using unabsorbed 2BB models. Accordingly, as argued in Paper I, one can compare 2BB parameters, inferred from observations (corrected for interstellar absorption and non-thermal radiation component), with the theoretical parameters of unabsorbed 2BB models. 

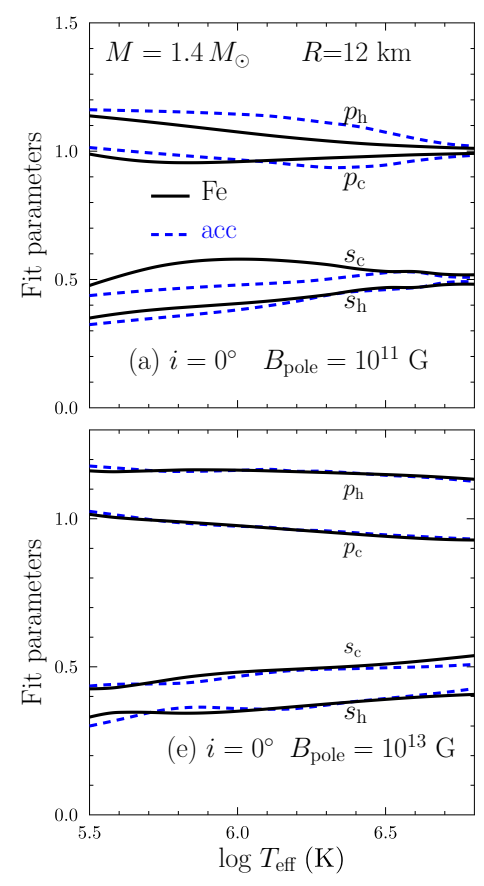
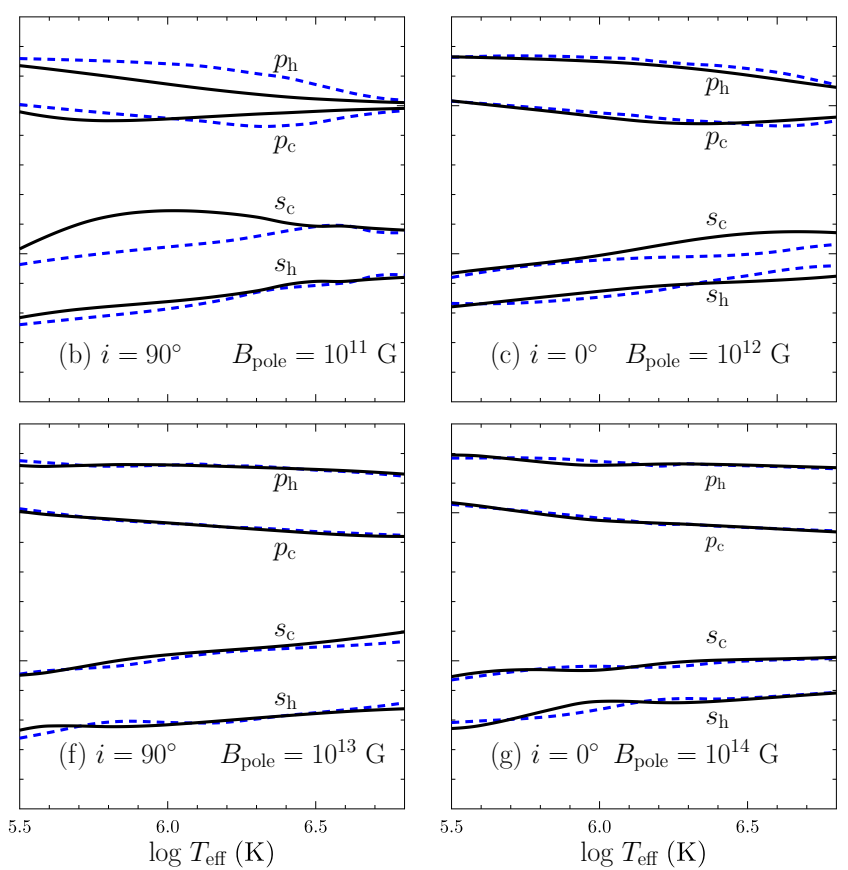
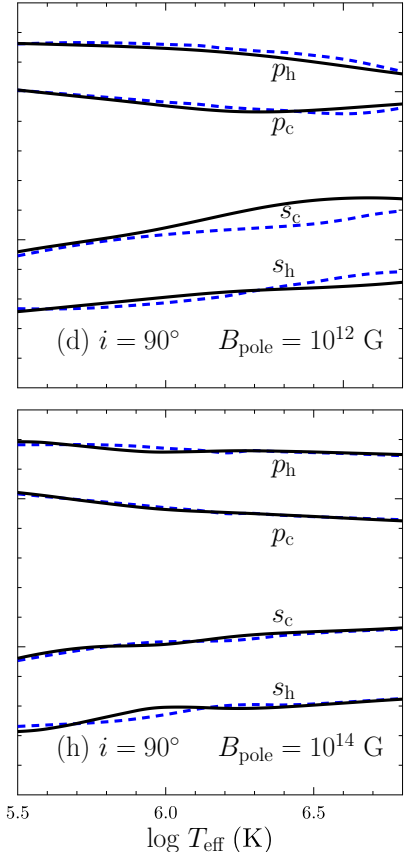

Figure 5. The $2 \mathrm{BB}$ fit parameters versus $\log T_{\text {eff }}$ for a $1.4 \mathrm{M}_{\odot}$ neutron star with $R=12 \mathrm{~km}$ and $B_{\text {pole }}=10^{11}, 10^{12}, 10^{13}$, or $10^{14} \mathrm{G}$ [panels $(\mathbf{a}-\mathbf{h})$ ] for polar $(i=0)$ and equator $\left(i=90^{\circ}\right)$ observations. Solid lines refer to the star with iron heat blanket, while dashed ones are for accreted heat blanket.

\section{Extra Heating of Magnetic Poles}

Section 4 describes the thermal emission of cooling neutron stars with dipole magnetic field near their surfaces, using the $T_{\mathrm{S}}$ model calculated in $[10,11]$. According to the theory, radiative spectral fluxes should be almost insensitive to the chemical composition of the heat blanketing envelopes; 2BB fits to their thermal X-ray spectral fluxes do not give the difference between the temperatures $T_{\text {effh }}$ and $T_{\text {effc }}$ of the hotter and colder BB components higher than $20 \%$ (for $B_{\text {pole }} \lesssim 10^{14} \mathrm{G}$ ).

A brief comparison with observations of isolated middle-aged neutron stars in Paper I shows that there are no reliable candidates for such objects at the moment; the difference between $T_{\text {effh }}$ and $T_{\text {effc }}$ is actually larger, at least $~ 50 \%$ for RX J1856.5-3754 [24,25], as a promising example.

Here, we propose a possible phenomenological extension of the discussed model, which may simplify the interpretation of observations of some sources. Specifically, let us assume the presence of additional heating of magnetic poles, which produces polar hotspots and raises the pole temperature. Such an extension has been used, for instance, in Paper I but assuming heating of both poles. Now we consider heating either of both poles or one of them. For simplicity, this heating is assumed to be axially symmetric. It does not violate the axial symmetry of the $T_{\mathrm{S}}$ distribution. Note that two equivalent hotspots do not destroy the initial symmetry of the north and south hemispheres, whereas one hotspot does destroy it. Possible physical justifications for extra heating are mentioned in Section 6.

Let $T_{\mathrm{s} 0}(\vartheta)$ be the basic effective surface temperature used in Section 3. We introduce a small phenomenological angle $\vartheta_{0}$ that deteminies the size of a hotspot. Following Paper I we assume that at $\vartheta<\vartheta_{0}$ (in the nothern hotspot),

$$
T_{\mathrm{s}}(\vartheta)=T_{\mathrm{s} 0}(\vartheta)\left[1+\delta \cos ^{2}\left(\frac{\pi \vartheta}{2 \vartheta_{0}}\right)\right] \text { at } \vartheta \leq \vartheta_{0}
$$

and $T_{\mathrm{s}}(\vartheta)=T_{\mathrm{s} 0}(\vartheta)$ outside the hotspot. The parameter $\delta$ specifies an extra temperature enhancement at the magnetic pole; the enhancement smoothly disappears as $\vartheta \rightarrow \vartheta_{0}$. This 
will be our model with one hotspot. Assuming a similar temperature enhancement near the second magnetic pole, we will get the model with two hot spots. The presence of spots renormalizes the total effective temperature $T_{\text {eff }}$, Equation (3).

Otherwise, the computation of spectral fluxes is the same as in Section 3. These fluxes can also be approximated by $2 \mathrm{BB}$ fits (6), and can be analyzed via phase-space maps. In some cases presented below the relative the fit accuracy becomes worse (reaching sometimes $\sim 10 \%$ ) but the fit remains robust. We will again take the star with $M=1.4 M_{\odot}$ and $R=12 \mathrm{~km}$. For certainty, we use the model of iron heat blanket and $\vartheta_{0}=10^{\circ}$.

\subsection{Extra Hotspots on Both Poles}

The left panel of Figure 6 demonstrates calculated spectral fluxes for $B_{\text {pole }}=10^{14} \mathrm{G}$ and seven values of $\log T_{\text {eff }}[\mathrm{K}]=5.6,5.8, \ldots, 6.8$ at $\delta=0.3$. Since these hotspots are identical, observations of the first and second poles $\left(i=0\right.$ and $\left.180^{\circ}\right)$ give the same fluxes. Such fluxes are shown by dashed lines. For comparison, the solid lines are calculated at $\delta=0$, in which case the extra hotspots are absent and the results of Section 3 apply. The dotted lines show the fluxes for non-magnetic star. One can see that the presence of hotspots enhances the spectral fluxes at high photon energies. This is expected: enhanced surface temperature of hotspots intensifies generation of high-energy radiation. The higher the $T_{\text {eff }}$, the larger the photon energies are affected. The solid and dotted lines are the same as in Figure 3c.
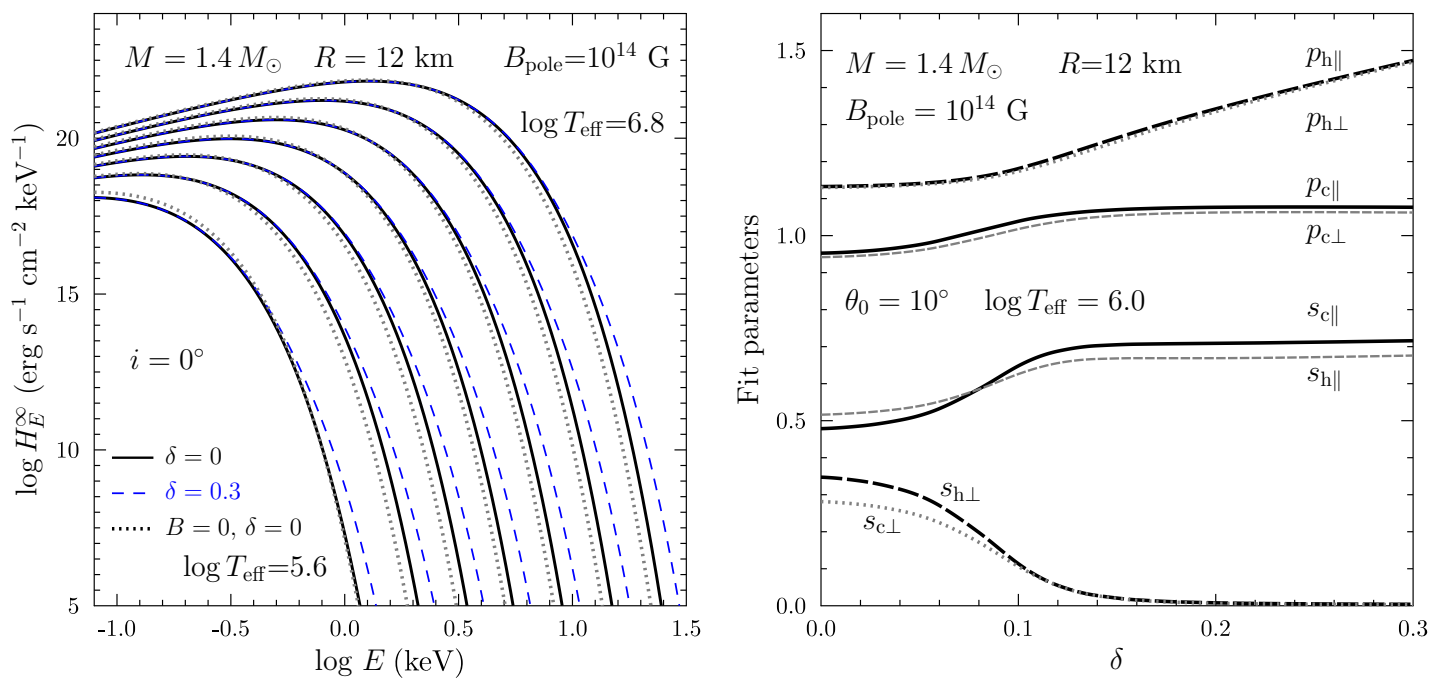

Figure 6. (Left): Thermal spectral fluxes observed from magnetic poles of a neutron star with $M=1.4 M_{\odot}$ and $R=12 \mathrm{~km}$ at $B_{\text {pole }}=10^{14} \mathrm{G}$ and $\log T_{\text {eff }}[\mathrm{K}]=5.6,5.8, \ldots, 6.6$. The solid lines are obtained without extra heating, while the dashed lines are for two extra hotspots of angular size $\vartheta_{0}=10^{\circ}$ on magnetic poles at the heating parameter $\delta=0.3$. Dots show $1 \mathrm{BB}$ model for a non-magnetic star to guide the eye. (Right): $2 \mathrm{BB}$ fit parameters for the same star with $T_{\text {eff }}=1 \mathrm{MK}$ possessing two polar hotspots of angular size $\vartheta_{0}=10^{\circ}$ on magnetic poles [see Equation (8)] versus extra relative surface temperature increase $\delta$ at the pole.

The right panel of Figure 6 shows the 2BB fit parameters versus $\delta$ for the same star with $T_{\text {eff }}=1$ MK. At $\delta=0$, the results naturally coincide with those in Figures $5 \mathrm{~g}$,h. However, with increasing $\delta$, the fit parameters become different. The temperature $T_{\text {effh }}$ of the hotter $\mathrm{BB}$ component and the effective emission surface area $s_{\mathrm{c}}$ of the colder component noticeably increase, whereas the emission surface area of the hotter component dramatically falls down. Even with really small hotspot temperature enhancement $\delta \leq 0.3$ (which gives the fraction of extra luminosity $\leq 1.4 \%$ ), one obtains an absolutely new phase-space portrait with $s_{\mathrm{h}} \ll s_{\mathrm{c}}$. Such $2 \mathrm{BB}$ fits have been often inferred from observations of cooling isolated neutron stars (see, e.g., Paper I); these sources are usually interpreted as neutron stars, which have small hotspots with noticeably enhanced temperature. 
Therefore, the theory with hotspots predicts (e.g., Paper I) two types of neutron stars, whose spectra can be approximated by the $2 \mathrm{BB}$ models. The first ones are those with smooth surface temperature distributions, created by nonuniform surface magnetic fields (as considered in Section 3) to be called spectral 2BB models of smooth magnetic atmospheres. The second sources are those with distinct hotspot BB component to be called 2BB with hotspots. Naturally, there is a smooth transition between them (for instance, by increasing $\delta$ in the right panel of Figure 6). It seems that the observations do not provide good candidates for the sources of the first type (Paper I), but there are some candidates for the sources of the second and intermediate types as we will outline later.

\subsection{Phase Resolved Spectroscopy in Case of Two Hotspots}

Let us present a few calculated lightcurves to illustrate an important problem of pulse fraction. For simplicity, we consider the star as an orthogonal rotator with the spin axis perpendicular to the line of sight. Figure 7 presents several lightcurves for a star with $B_{\text {pole }}=10^{14} \mathrm{G}$ versus phase angle $\theta$. The lightcurves are normalized by $H_{E}(0)$ (the spectral flux for pole observations at given energy $E$ ). The displayed ratio of redshifted or non-redshifted fluxes is the same at a given $E$, so that we drop the symbol $\infty$. Note that the normalization flux $H_{E}(0)$ strongly depends on energy by itself. Panel (a) corresponds to $\delta=0$, in which case the hotspots are actually absent and we have the emission from the smooth magnetic atmosphere. In case (b) the hotspots with $\delta=0.3$ are available and strongly affect the lightcurves.
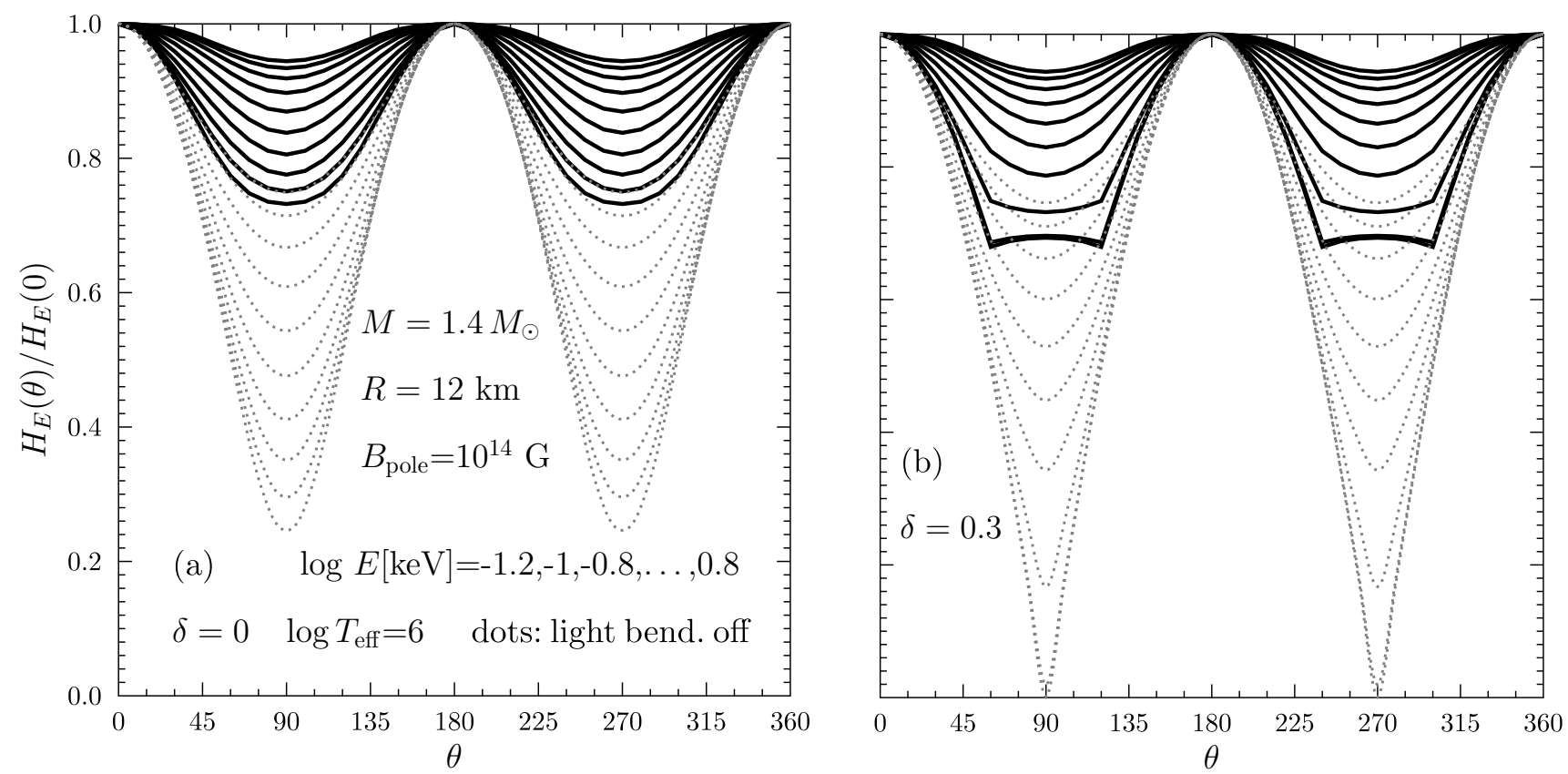

Figure 7. Theoretical lightcurves, normalized to pole observations, versus phase angle $\theta$ for a neutron star with $T_{\text {eff }}$ $=1 \mathrm{MK}$ and $B_{\text {pole }}=10^{14} \mathrm{G} ;(\mathbf{a}) \delta=0$ (no extra pole heating) and $(\mathbf{b}) \delta=0.3$. Solid curves are for photon energies $\log E[\mathrm{keV}]=-1,-0.8, \ldots, 0.8$ (from top to bottom). The two lowest curves on panel (b) (with highest $E$ ) almost coincide. Dotted curves show the same but neglecting gravitational light bending. The star is assumed to be orthogonal rotator with the spin axis perperndicular to the line of sight.

Solid lines on each panel in Figure 7 present the lightcurves at 10 energies (from top to bottom), $\log E[\mathrm{keV}]=-1,-0.8, \ldots, 0.8$. The higher $E$, the stronger phase variations. The last two lines on panel (b) almost coincide. The lightcurves on panel (a) are seen to be smooth. The curves on panel (b) at $E \lesssim 1 \mathrm{keV}$ are although smooth and resemble those on panel (a). However, at higher energies the lightcurves (b) have shapes with erased dips at nearly equator observations [and then Equation (7) becomes inaccurate although 
Equation (6) works well]. These curves are typical for lightcurves produced by antipodal point sources on the neutron star surface; see, e.g., Figure 4 in [19] or Figure 6 in [21]. Clearly, at high energies the star emits from the poles, the hottest places on the surface. Nevertheless, the pulse fraction remains not too high $(\leq 30 \%)$ in both cases (a) and (b) even at the highest taken energy $E \approx 6.3 \mathrm{keV}$, at which thermal spectral flux becomes typically very low by itself. Therefore, the enhancement of thermal emission by extra heating of two magnetic poles does not lead to sizeable pulse fractions.

The dotted lines on Figure 7 show the same lightcurves as the solid lines but calculated neglecting gravitational bending of light rays. One sees that without the light bending the pulse fraction would be much stronger (as is well known; see, for example, [8]). The difference of solid and dotted curves on Figure 7 has simple explanation. Without light bending, the equator observations $\left(\theta=90^{\circ}\right.$ or $\left.270^{\circ}\right)$ would collect more light emitted from the cold equator. This would lower the observed emission and produce stronger dips of the lightcurves.

\subsection{Extra Hotspot on One Pole}

Finally, consider the case of a single hotspot, which we put at the north pole. This case is different from the previous one.

The left panel of Figure 8 presents spectral fluxes calculated for the same values of $T_{\text {eff }}$ and $B_{\text {eff }}$ as in Figure 6. The dashed lines are for the north-pole observations $(i=0)$ at $\delta=0.3$. These fluxes are the same as in Figure 6: the observer sees a large fraction of the surface (mainly the northern hemisphere); the southern polar region is unseen.

The solid line in the left panel of Figure 8 refers to the same case of $\delta=0.3$ but the star is observed from the south pole $\left(i=180^{\circ}\right)$. Then the observer cannot see the hotspot, so the line exactly coincides with the solid line in Figure 3c. The dotted line in Figure 8 is for a non-magnetic star (as in Figures 3c and 6). Now, with only one extra heated pole, the difference between observations of the north and south poles is pronounced.
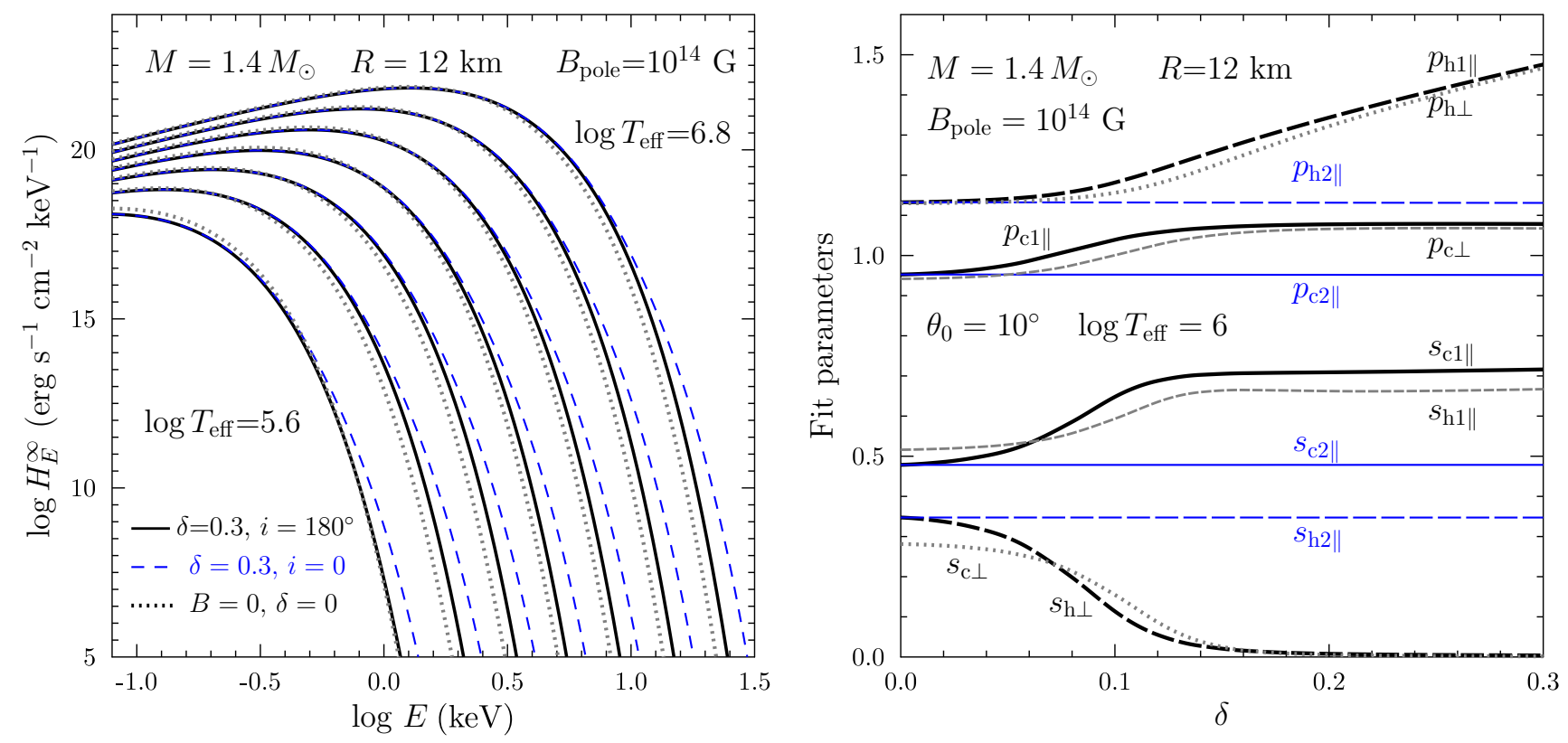

Figure 8. (Left): Theoretical spectral fluxes for a neutron star with $M=1.4 M_{\odot}, R=12 \mathrm{~km}, B_{\text {pole }}=10^{14} \mathrm{G}$, different $T_{\text {eff }}$ and one extra hotspot on one magnetic pole at the extra heating parameter $\delta=0.3$. The solid and dashed curves are for non-heated $\left(i=180^{\circ}\right)$ and heated pole $(i=0)$ observations, respectively. (Right): Maps of fit parameters versus $\delta$ for this star at $T_{\text {eff }}=1 \mathrm{MK}$. The thick solid and gray curves are for the north-pole and equator observations, respectively. Thin horizontal curves are for the south-pole observations. 
The right panel of Figure 8 presents the maps of 2BB fit parameters versus $\delta$ for the same star at $T_{\text {eff }}=1 \mathrm{MK}$ for the north-pole, south-pole, and equator observations (to be compared with Figure 6). The maps the for north-pole and equator observations are nearly the same as those in Figure 6, but the maps for the south-pole observations are plain. Corresponding fit parameters are independent of $\delta$ just because the observer cannot see the emission from the hotspot (which is the only emission which increases with $\delta$ ).

\subsection{Phase Resolved Spectroscopy in Case of One Hotspot}

Phase-resolved lightcurves are plotted in Figures 9 and 10. They are naturally different from the case of two extra heated magnetic poles. Figure 9 is analogous to Figure 7 and shows the light curves at the same $T_{\text {eff }}, B_{\text {pole, }}$ and extra heating parameter $\delta=0.3$ (a) and $\delta=0.14$ (b). With one hotspot, the fractions of extra luminosities are $0.7 \%$ (a) and $0.3 \%$ (b). The phase-space variations are similar to those in Figure 7 only at low photon energies, in which case the extra polar heating is almost unnoticeable and the lightcurves have two shallow dips over one rotation period. However with growing $E$, the extra heating becomes important and increases the depth and width of the dips (such shapes are known to be produced by point sources on neutron star surface, e.g., Figure 4 in [19]). At $E \gtrsim 4 \mathrm{keV}$ in Figure 9a the pulse fraction becomes close to $100 \%$. In that case the observer would see only one, but very pronounced dip over one rotation, because the extra hotspot would be poorly visible in a wide range of $\theta$ with the center at $\theta=180^{\circ}$. In Figure $9 \mathrm{~b}$ the heating is weaker, the dips are smaller, but nevertheless they are much larger than for two heated spots at $\delta=0.3$ in Figure 7. Evidently, one extra-heated magnetic pole is much more profitable than two, for producing large pulse fractions.
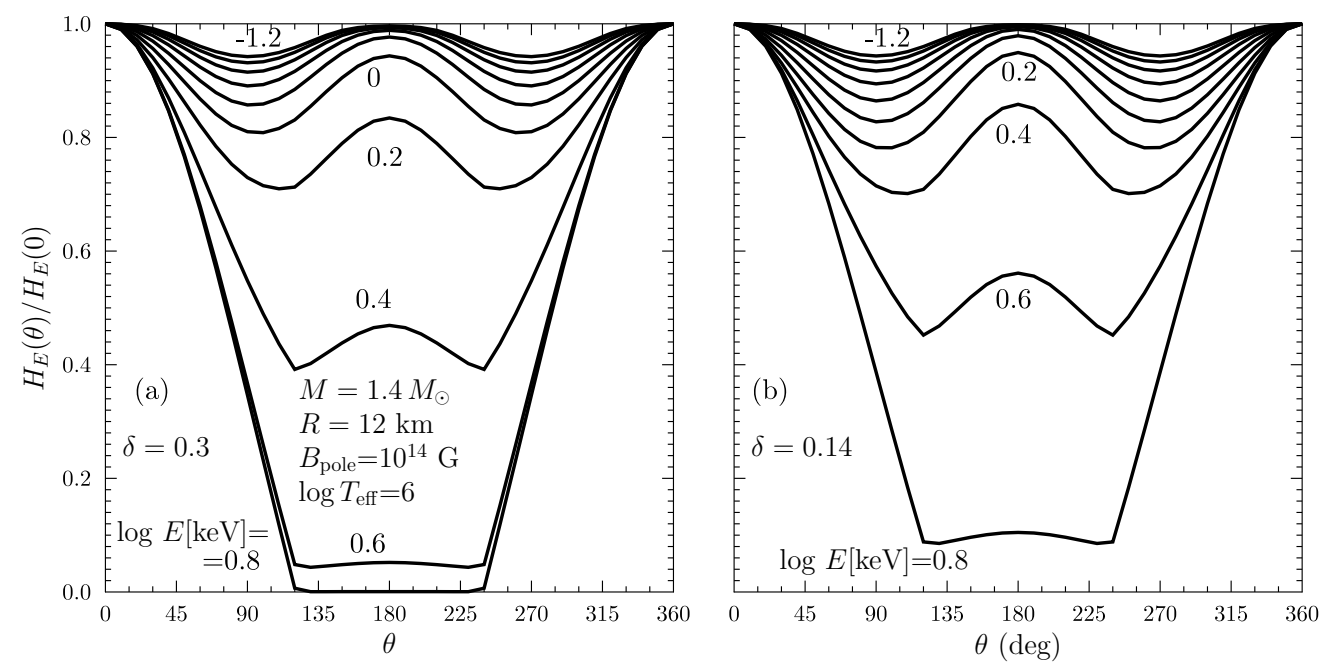

Figure 9. Theoretical lightcurves for a neutron star with $M=1.4 M_{\odot}, R=12 \mathrm{~km}, T_{\text {eff }}=1 \mathrm{MK}$ and $B_{\text {pole }}=10^{14} \mathrm{G}$ with an extra hotspot of angular size $\vartheta_{0}=10^{\circ}$ on one magnetic pole versus phase angle $\theta$ at extra heating parameter $\delta=0.3(\mathbf{a})$ and $0.14(\mathbf{b})$. The curves are for photon energies $\log E[\mathrm{keV}]=-1,-0.8, \ldots, 0.8$. See the text for details.

Figure 10 is plotted for $B_{\text {pole }}=10^{11} \mathrm{G}$ to illustrate the effects of $B_{\text {pole }}$ on the pulse fraction. Figure 10a presents the normalized lightcurves for the star with $\delta=0.3$. Now the pulse fraction is lower than at $B_{\text {pole }}=10^{14} \mathrm{G}$. Nevertheless, it remains much higher than it would be if the additional heating was switched on at both poles. Figure $10 \mathrm{~b}$ demonstrates that at lower $\delta=0.14$ at $B_{\text {pole }}=10^{11} \mathrm{G}$ the pulse fraction is weaker affected by the extra heating but nevertheless reaches about $70 \%$ at $E \sim 6 \mathrm{keV}$. 

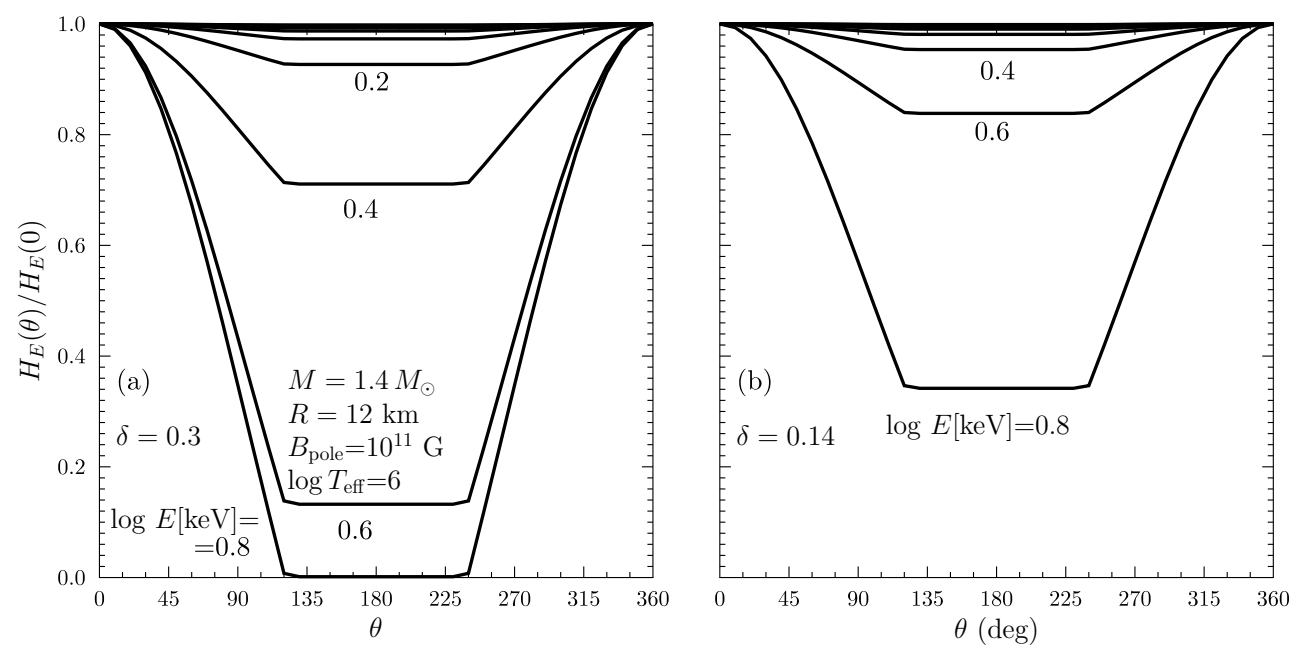

Figure 10. Same as in Figure 9 but for $B_{\text {pole }}=10^{11} \mathrm{G}$.

\section{Discussion and Conclusions}

Several isolated middle-aged neutron stars which radiation spectra have been (or can be) fitted by 2BB models have been selected in Paper I, based on the recent catalog [26] presented also on the Web: www.ioffe.ru/astro/NSG/thermal/cooldat.html, accessed on 17 October 2021.

These can be cooling neutron stars with dipole magnetic fields and additionally heated polar caps. This selection is illustrative (does not pretend to be complete).

1. XMMU J172054.5-372652 is a neutron star that is probably associated with the SNR G350.1-0.3 [27]; there is no direct evidence of pulsations. Potekhin et al. [26] used archival Chandra data and fitted the X-ray spectrum with a neutron star (NSX) atmosphere model assuming $M=1.4 M_{\odot}$ and $R=13 \mathrm{~km}$. They obtained $T_{\text {eff }} \approx 2 \mathrm{MK}$, but did not perform two-component fits which would be interesting.

2. PSR B1055-52 (J1057-5226) is a moderately magnetized middle-aged pulsar. Its characteristic effective magnetic field, reported in the ATNF pulsar catalog [28], is $B_{\text {eff }}=1.1 \times 10^{12} \mathrm{G}$. Potekhin et al. [26] present the value $k_{\mathrm{B}} T_{\mathrm{eff}}^{\infty} \approx 70 \mathrm{eV}\left(T_{\mathrm{eff}}^{\infty} \approx 0.8 \mathrm{MK}\right)$ based on the 2BB spectral fit by De Luca et al. [29]; the fit includes also a power-law (PL) non-thermal radiation component. Potekhin et al. [26] have corrected the results of [29] for the distance estimate to the source made by Mignani et al. [30] and reported $T_{\text {effh }} / T_{\text {effc }} \sim 2.3$ with $s_{\mathrm{h}} \ll s_{\mathrm{C}}$. It seems worth to try to explain these data with a $2 \mathrm{BB}$ model containing hotspots.

3. PSR J1740+1000 possesses the magnetic field $B_{\text {eff }}=1.8 \times 10^{12} \mathrm{G}$. The $2 \mathrm{BB}$ spectral fit was performed in [31]. With the same version of the fit as selected in [26], one has $k_{\mathrm{B}} T_{\mathrm{eff}}^{\infty} \approx 70 \mathrm{eV}\left(T_{\mathrm{eff}}^{\infty} \approx 0.8 \mathrm{MK}\right), T_{\mathrm{effh}} / T_{\mathrm{effc}} \sim 2.8$ and $s_{\mathrm{h}} \ll s_{\mathrm{c}}$, with the same conclusion as for the PSR B1055-52.

4. PSR B1823-13 (J1826-1334), located in the SNR G18.0-00.7, has $B_{\text {eff }}=2.8 \times 10^{12}$ G. Its $\mathrm{X}$-ray emission is mainly non-thermal [32,33] but has some thermal component. The $1 \mathrm{BB}+\mathrm{PL}$ fit gives the radius of thermally emitting region $R_{\mathrm{eff}}^{\infty} \approx 5 \mathrm{~km}$, smaller than the expected radius of a neutron star. Adding the second BB component is statistically insignificant with the present data, but might be possible in the future.

5. RX J1605.3+3249 (RBS 1556) is a neutron star studied by many authors (e.g., [34-38]) with contradictory conclusions on its properties (as detailed in [26]). There is no solid evidence of stellar rotation [37]. Its X-ray emission has been analyzed using BB fits and neutron star atmosphere models. Recently Pires et al. [37] analysed the XMMNewton observations and Malacaria et al. [38] jointly analysed the NICER and XMMNewton data. These teams improved 2BB fits by adding a broad Gaussian absorption line, in which case they got $k_{\mathrm{B}} T_{\text {effc }}^{\infty} \sim 60 \mathrm{eV}\left(T_{\mathrm{effc}}^{\infty} \sim 0.7 \mathrm{MK}\right)$, and $T_{\text {effh }} / T_{\text {effc }} \sim 2$, a good opportunity to assume a dipole magnetic field and additionally heated poles. 
6. $\mathrm{RX}$ J1856.5-3754 is a neutron star with nearly thermal spectrum. It was discovered by Walter et al. [39]. It is rotating with the spin period of $\sim 7 \mathrm{~s}$; the effective magnetic field is $B_{\text {eff }} \sim 1.5 \times 10^{13} \mathrm{G}$; magnetic properties are highly debated (e.g., $[18,40]$ and references therein). The spectrum has been measured in a wide energy range, including X-rays, optical and radio, and interpreted with many spectral models, particularly, with the model of thin partially ionized magnetized hydrogen atmosphere on top of solidified iron surface (see, e.g., $[5,41]$ ). Note alternative $2 \mathrm{BB}, 2 \mathrm{BB}+\mathrm{PL}$, and 3BB fits constructed by [24] and [25]. The 2BB fits give $k_{\mathrm{B}} T_{\mathrm{effc}}^{\infty} \sim 40 \mathrm{eV}\left(T_{\mathrm{effc}}^{\infty} \approx 0.46 \mathrm{MK}\right)$, $T_{\text {effh }} / T_{\text {effc }} \sim 1.6, R_{\text {effh }} \sim 0.5 R$ and $R_{\text {effc }} \sim R$; they seem closer to the $2 \mathrm{BB}$ spectral models with smooth magnetic atmosphere, than 2BB fits for other sources. Extra heating of magnetic poles would simplify this interpretation.

This brief analysis does not reveal any good candidate which would belong to the family of neutron stars with dipole magnetic fields and smooth surface temperature distribution (Section 4). This does not mean that such neutron stars do not exist; it can be difficult to identify them because their spectra are close to 1BB spectrum. To interpret the above sources one needs to complicate the model, for instance, by introducing extra heating of magnetic poles.

Now let us summarize our results. Following Paper I we have studied simple models (Section 3) of thermal spectra emitted from surfaces of isolated neutron stars with dipole surface magnetic fields $10^{11} \lesssim B_{\text {pole }} \lesssim 10^{14} \mathrm{G}$. Such fields make the surface temperature distribution noticeably non-uniform. The model assumes BB emission with a local temperature from any surface element.

In Section 4 we have shown that the spectral X-ray fluxes emitted from such neutron stars are almost the same for heat blanketing envelopes composed of iron and fully accreted matter (at the same average effective surface temperatures $T_{\text {eff }}$ ). By measuring the spectral fluxes and $T_{\text {eff }}$, it would be difficult to infer composition of their heat blanketing envelopes, although this composition can noticeably affect neutron star cooling (e.g., [14] and references therein). In the presence of fully accreted matter, the fluxes are accurately fitted by 2BB models (as in Paper I for the iron heat blankets). At $M \sim 1.4 M_{\odot}$ and $R \sim 12 \mathrm{~km}$, the ratio $T_{\text {effh }} / T_{\text {effc }}$ of effective temperatures of the hotter to colder BB components cannot be essentially larger than $\sim 1.2$. For less compact stars, with smaller $M / R$, this value can be somewhat higher (see e.g., [8], Paper I). If this ratio is larger from observations, then the model of Sections 3 and 4 cannot explain the data.

In Section 5, following Paper I, we have extended the model by assuming sufficiently weak extra heating of one or both magnetic poles. Increasing extra relative temperature rise $\delta$ at the pole(s), does not greatly violate the accuracy of the 2BB spectral approximation, but allows one to obtain larger $T_{\text {effh }} / T_{\text {effc }}$. Also, it affects the pulse fraction, which increases with $B_{\text {pole }}$ and photon energy $E$. The pulse fraction can be very high if one magnetic pole is additionally heated, while the other is not.

Let us stress, that there have been many other studies of thermal emission from magnetized neutron stars. The emission produced by anisotropic surface temperature distribution of the star with dipole magnetic fields was analyzed by Page [8]. Quadrupole magnetic field components were added in [9]. A possible presence of toroidal field component in the neutron star crust was studied in $[12,13]$. Also, there were studies of thermal emission during magnetic field evolution in neutron stars (e.g., $[18,40])$. These works give a variety of magnetic fields configurations, surface temperature distributions, and phase-resolved spectra of neutron stars to be compared with observations and determine simultaneously both, the surface temperature distribution and magnetic field geometry.

Introducing some extra heating of magnetic poles, we are actually doing the same. Note that hot spots can be produced by pulsar mechanism. Also, the toroidal crustal magnetic fields can significantly widen the cold equatorial belt, creating relatively stronger heated poles [12], which can mimic hotspots. At the first step it might be simpler to introduce phenomenological polar heating, that can be different on the two poles, and to determine the parameters of the extra heating (the $T_{\mathrm{s}}$ distribution) by varying the param- 
eters like $\delta$ and extra temperature profile [Equation (8)] at both poles. This would be a simple and physically transparent model of surface temperature distribution. At the next step, one could infer (constrain) the magnetic field geometry. This method can be easily extended to different $T_{\mathrm{s}}$ distributions, not necessarily axially symmetric.

Another very important direction would be to go beyond the approximation of local BB emission from any surface patch, and use more realistic emission models (with spectral features and anisotropic radiation, as well as with account of polarization effects, for example, $[42,43])$.

Funding: This research was partly (in analyzing the case of accreted matter) supported by the Russian Science Foundation, grant 19-12-00133.

Institutional Review Board Statement: Not applicable.

Informed Consent Statement: Not applicable.

Data Availability Statement: The data underlying this article will be shared on reasonable request to the corresponding author.

Acknowledgments: I am grateful to Oleg Gnedin for providing me photos of his Father.

Conflicts of Interest: The author declares no conflict of interest.

\section{Abbreviations}

The following abbreviations are used in this manuscript:

BB blackbody

\section{References}

1. Gnedin, Y.N.; Dolginov, A.Z.; Tsygan, A.I. Possible mechanism of formation of the spectra of cosmic X-ray sources. Sov. J. Exp. Theor. Phys. Lett. 1969, 10, 283-285.

2. Gnedin, Y.N.; Pavlov, G.G. The transfer equations for normal waves and radiation polarization in an anisotropic medium. Sov. J. Exp. Theor. Phys. 1974, 38, 903-908.

3. Gnedin, Y.N.; Sunyaev, R.A. Polarization of optical and X-radiation from compact thermal sources with magnetic field. Astron. Astrophys. 1974, 36, 379-394.

4. Gnedin, Y.N.; Pavlov, G.G.; Tsygan, A.I. Photoeffect in strong magnetic fields and x-ray emission from neutron stars. Sov. J. Exp. Theor. Phys. 1974, 39, 201.

5. Potekhin, A.Y. Atmospheres and radiating surfaces of neutron stars. Phys. Uspekhi 2014, 57, 735. [CrossRef]

6. Potekhin, A.Y.; De Luca, A.; Pons, J.A. Neutron Stars - Thermal Emitters. Space Sci. Rev. 2015, 191, 171-206. [CrossRef]

7. Greenstein, G.; Hartke, G.J. Pulselike character of blackbody radiation from neutron stars. Astrophys. J. 1983, $271,283-293$. [CrossRef]

8. Page, D. Surface temperature of a magnetized neutron star and interpretation of the ROSAT data. 1: Dipole fields. Astrophys. J. 1995, 442, 273-285. [CrossRef]

9. Page, D.; Sarmiento, A. Surface Temperature of a Magnetized Neutron Star and Interpretation of the ROSAT Data. II. Astrophys. J. 1996, 473, 1067. [CrossRef]

10. Potekhin, A.Y.; Yakovlev, D.G. Thermal structure and cooling of neutron stars with magnetized envelopes. Astron. Astrophys. 2001, 374, 213-226. [CrossRef]

11. Potekhin, A.Y.; Yakovlev, D.G.; Chabrier, G.; Gnedin, O.Y. Thermal Structure and Cooling of Superfluid Neutron Stars with Accreted Magnetized Envelopes. Astrophys. J. 2003, 594, 404-418. [CrossRef]

12. Geppert, U.; Küker, M.; Page, D. Temperature distribution in magnetized neutron star crusts. II. The effect of a strong toroidal component. Astron. Astrophys. 2006, 457, 937-947. [CrossRef]

13. Zane, S.; Turolla, R. Unveiling the thermal and magnetic map of neutron star surfaces though their X-ray emission: Method and light-curve analysis. MNRAS 2006, 366, 727-738. [CrossRef]

14. Beznogov, M.V.; Potekhin, A.Y.; Yakovlev D.G. Heat blanketing envelopes of neutron stars. Phys. Rep. 2021, 919, 1-68. [CrossRef]

15. Yakovlev, D.G. Two-blackbody portraits of radiation from magnetized neutron stars. MNRAS 2021, 506, 4593-4602. [CrossRef]

16. Zavlin, V.E.; Pavlov, G.G. Modeling Neutron Star Atmospheres. In Neutron Stars, Pulsars, and Supernova Remnants; Becker, W., Lesch, H., Trümper, J., Eds., Max-Plank-Institut für extraterrestrische Physik: Garching bei München, Germany, $2002 ;$ p. 263.

17. González Caniulef, D.; Zane, S.; Taverna, R.; Turolla, R.; Wu, K. Polarized thermal emission from X-ray dim isolated neutron stars: the case of RX J1856.5-3754. MNRAS 2016, 459, 3585-3595. [CrossRef] 
18. De Grandis, D.; Taverna, R.; Turolla, R.; Gnarini, A.; Popov, S.B.; Zane, S.; Wood, T.S. X-ray Emission from Isolated Neutron Stars revisited: 3D magnetothermal simulations. Astrophys. J. 2021, 914, 118. [CrossRef]

19. Beloborodov, A.M. Gravitational Bending of Light Near Compact Objects. Astrophys. J. Lett. 2002, 566, L85-L88. [CrossRef]

20. Poutanen, J.; Gierliński, M. On the nature of the X-ray emission from the accreting millisecond pulsar SAX J1808.4-3658. MNRAS 2003, 343, 1301-1311. [CrossRef]

21. Poutanen, J. Accurate analytic formula for light bending in Schwarzschild metric. Astron. Astrophys. 2020, 640, A24. [CrossRef]

22. Potekhin, A.Y.; Chabrier, G.; Yakovlev, D.G. Internal temperatures and cooling of neutron stars with accreted envelopes. Astron. Astrophys. 1997, 323, 415-428.

23. Haensel, P.; Potekhin, A.Y.; Yakovlev, D.G. Neutron Stars. 1. Equation of State and Structure; Astrophysics and Space Science Library; Springer: New York, NY, USA, 2007; Volume 326.

24. Sartore, N.; Tiengo, A.; Mereghetti, S.; De Luca, A.; Turolla, R.; Haberl, F. Spectral monitoring of RX J1856.5-3754 with XMM-Newton. Analysis of EPIC-pn data. Astron. Astrophys. 2012, 541, A66. [CrossRef]

25. Yoneyama, T.; Hayashida, K.; Nakajima, H.; Inoue, S.; Tsunemi, H. Discovery of a keV-X-ray excess in RX J1856.5-3754. Publ. Astron. Soc. Jpn. 2017, 69, 50. [CrossRef]

26. Potekhin, A.Y.; Zyuzin, D.A.; Yakovlev, D.G.; Beznogov, M.V.; Shibanov, Y.A. Thermal luminosities of cooling neutron stars. MNRAS 2020, 496, 5052-5071. [CrossRef]

27. Gaensler, B.M.; Tanna, A.; Slane, P.O.; Brogan, C.L.; Gelfand, J.D.; McClure-Griffiths, N.M.; Camilo, F.; Ng, C.Y.; Miller, J.M. The (Re-)Discovery of G350.1-0.3: A Young, Luminous Supernova Remnant and Its Neutron Star. Astrophys. J. Lett. 2008, 680, L37. [CrossRef]

28. Manchester, R.N.; Hobbs, G.B.; Teoh, A.; Hobbs, M. The Australia Telescope National Facility Pulsar Catalogue. Astron. J. 2005, 129, 1993-2006. [CrossRef]

29. De Luca, A.; Caraveo, P.A.; Mereghetti, S.; Negroni, M.; Bignami, G.F. On the Polar Caps of the Three Musketeers. Astrophys. J. 2005, 623, 1051-1069. [CrossRef]

30. Mignani, R.P.; Pavlov, G.G.; Kargaltsev, O. Optical-Ultraviolet Spectrum and Proper Motion of the Middle-aged Pulsar B1055-52. Astrophys. J. 2010, 720, 1635-1643. [CrossRef]

31. Kargaltsev, O.; Durant, M.; Misanovic, Z.; Pavlov, G.G. Absorption Features in the X-ray Spectrum of an Ordinary Radio Pulsar. Science 2012, 337, 946. [CrossRef]

32. Pavlov, G.G.; Kargaltsev, O.; Brisken, W.F. Chandra Observation of PSR B1823-13 and Its Pulsar Wind Nebula. Astrophys. J. 2008, 675, 683-694. [CrossRef]

33. Zhu, W.W.; Kaspi, V.M.; McLaughlin, M.A.; Pavlov, G.G.; Ng, C.Y.; Manchester, R.N.; Gaensler, B.M.; Woods, P.M. Chandra Observations of the High-magnetic-field Radio Pulsar J1718-3718. Astrophys. J. 2011, 734, 44. [CrossRef]

34. Motch, C.; Sekiguchi, K.; Haberl, F.; Zavlin, V.E.; Schwope, A.; Pakull, M.W. The proper motion of the isolated neutron star J1605.3+3249 . Astron. Astrophys. 2005, 429, 257-265. [CrossRef]

35. Posselt, B.; Popov, S.B.; Haberl, F.; Trümper, J.; Turolla, R.; Neuhäuser, R. The Magnificent Seven in the dusty prairie. Astrophys. Space Sci. 2007, 308, 171-179. [CrossRef]

36. Tetzlaff, N.; Schmidt, J.G.; Hohle, M.M.; Neuhäuser, R. Neutron Stars From Young Nearby Associations: The Origin of RX J1605.3+3249. Publ. Astron. Soc. Aust. 2012, 29, 98-108. [CrossRef]

37. Pires, A.M.; Schwope, A.D.; Haberl, F.; Zavlin, V.E.; Motch, C.; Zane, S. A deep XMM-Newton look on the thermally emitting isolated neutron star RX J1605.3+3249. Astron. Astrophys. 2019, 623, A73. [CrossRef]

38. Malacaria, C.; Bogdanov, S.; Ho, W.C.G.; Enoto, T.; Ray, P.S.; Arzoumanian, Z.; Cazeau, T.; Gendreau, K.C.; Guillot, S.; Güver, T.; et al. A Joint NICER and XMM-Newton View of the "Magnificent" Thermally Emitting X-Ray Isolated Neutron Star RX J1605.3+3249. Astrophys. J. 2019, 880, 74. [CrossRef]

39. Walter, F.M.; Wolk, S.J.; Neuhäuser, R. Discovery of a nearby isolated neutron star. Nature 1996, 379, 233-235. [CrossRef]

40. Popov, S.B.; Taverna, R.; Turolla, R. Probing the surface magnetic field structure in RX J1856.5-3754. MNRAS 2017, 464, 4390-4398. [CrossRef]

41. Ho, W.C.G.; Kaplan, D.L.; Chang, P.; van Adelsberg, M.; Potekhin, A.Y. Magnetic hydrogen atmosphere models and the neutron star RX J1856.5-3754. MNRAS 2007, 375, 821-830. [CrossRef]

42. Ho, W.C.G.; Potekhin, A.Y.; Chabrier, G. Model X-Ray Spectra of Magnetic Neutron Stars with Hydrogen Atmospheres. Astrophys. J. Suppl. 2008, 178, 102-109. [CrossRef]

43. Zyuzin, D.A.; Karpova, A.V.; Shibanov, Y.A.; Potekhin, A.Y.; Suleimanov, V.F. Middle aged $\gamma$-ray pulsar J1957+5033 in X-rays: pulsations, thermal emission, and nebula. MNRAS 2021, 501, 4998-5011. [CrossRef] 